\title{
MAPPING CONVECTION IN THE MANTLE
}

\author{
Toshiro Tanimoto and Don L. Anderson
}

Seismological Laboratory, California Institute of Technology, Pasadena, CA 91125

\begin{abstract}
Long-period (100-250 sec.) Love and Rayleigh waves are used to map heterogeneity and azimuthal anisotropy in the upper mantle. Spherical harmonic descriptions of anisotropy up to $\ell=\mathrm{m}=3$ and $2 \theta$ are derived. Azimuthal anisotropy obtains values as high as $1 \frac{1}{2} \%$. There is good correlation of fast Rayleigh wave directions with upper mantle return flow directions derived from kinematic considerations. This is consistent with the a-axis of olivine being aligned in the flow direction. The main differences between the flow models and the Rayleigh wave azimuthal variation maps occur in the vicinity of hotspots. Hawaii, for example, appears to perturb the return flow. There is strong correlation of the geoid and surface wave velocity at $\ell=4$ and 5 . Slow regions at this scale are associated with geoid highs and high heat flow, consistent with upwelling convective flow or with isostatically compensated regions of low density. The correlation of azimuthal anisotropy with upper mantle return flow directions, rather than with plate directions, suggests that part of the return flow is in the upper mantle and this, in turn, implies a low viscosity channel.
\end{abstract}

\section{Introduction}

Understanding convection in the mantle is one of the most important goals of geophysics. To date, the geoid, heat flow, and elevation have been the main constraints on theoretical models of convection. However, we still have a poor understanding of the scale of mantle convection and the driving mechanism of plate tectonics. Three-dimensional global maps of seismic velocity anomalies are starting to become available [Nakanishi and Anderson, 1984; Masters et al, 1982] and promise to help constrain the nature of the flow. Seismic techniques can be used to map anisotropy as well as heterogeneity and, in principle, can determine crystal orientation. Global maps of transverse isotropy have previously been used to map regions of upwelling and downwelling in the mantle [Nataf el al., 1984]. We report here the results of a first attempt to obtain and interpret global maps of azimuthal anisotropy from long-period surface waves. The method we use is described by Tanimoto and Anderson, [1983]. The data set is phase velocity over about 560 paths for Rayleigh waves and 380 paths for Love waves in the period range 100 to 250 seconds. These waves give a good sampling of velocities in the outer $400 \mathrm{~km}$ of the mantle. The data are simultaneously inverted for heterogeneity and azimuthal anisotropy. For heterogeneity we use a spherical harmonic representation including terms up to $\ell=m=6$. For anisotropy we solve for $2 \theta$ terms and display results for $\ell=m=1$ to 3 .

\section{Copyright 1984 by the American Geophysical Union.}

Paper number 4L0373.

0094-8276/84/004L-0373\$03.00

\section{Heterogeneity}

Figure 1 shows maps of Rayleigh wave phase velocities with $\ell$ max $=6$ for a period of $200 \mathrm{sec}$. These waves are most sensitive to SV-velocities between about 150 and $400 \mathrm{~km}$. Regions of faster than average velocity occur in the western $\mathrm{Pa}$ cific, the western part of the African plate, Australia southern Indian Ocean, part of the south Atlantic, northeastern North America - western north Atlantic, and northern Europe. These are all relative geoid lows confirming the correlations found by Nakanishi and Anderson [1984]. Dense regions of the mantle that are in isostatic equilibrium also generate geoid lows [Haxby and Turcotte, 1978; Hager, 1983]. Dense regions in dynamic equilibrium generate geoid lows if the viscosity does not increase too rapidly with depth [Hager, 1984]. High density and high velocity are both consistent with cold mantle. Geoid highs occur near Tonga-Fiji, Andes, Borneo, Red Sea, Alaska, northern Atlantic , and southern Indian Ocean. These are generally slow regions of the mantle and are therefore presumably hot. Uprising plumes would be consistent with these observations. The upward deformation of boundaries counteracts the low density associated with the buoyant material and, for uniform viscosity, the net result is a geoid high (Hager, 1983). An isostatically compensated column of low density material also generates a geoid high because of the elevation of the surface. Correlation coefficients between phase velocity and the geoid are shown in Fig. 2. Love waves for periods between 100 and 200 $s$ generally have a strong negative correlation (geoid highs correlate with slow velocity) for $\ell=4,5,6$. Rayleigh waves generally correlate well with the geoid for $\ell=6$ and, at some periods with $\ell=5$ (Fig. 1 and Nakanishi and Anderson, 1984).

Hager [1984] found an excellent correlation between the observed geoid and the 'slab geoid' for $\ell=4,5$ and 7-9 and weak correlation at $\ell=6$. The slab geoid does not explain the geoid lows in Siberia, Canadian shield - western Atlantic, and the South Atlantic or the geoid highs in the south Indian Ocean, the Red Sea, and the North Atlantic. These are highvelocity and low-velocity regions, respectively. The slab-geoid correlation can be understood in terms of a large amount of dynamically supported dense material either in the upper mantle or in both the upper and lower mantles in the vicinity of active subduction zones [Hager, 1984]. The surface wave - geoid correlation, which apparently explains the missing $\ell=6$ component of the slab geoid, however, must be due, at least in part, to an anomalous mass distribution in the shallow mantle. These results suggest that features of the geoid having wavelengths of about 4,000 to $10,000 \mathrm{~km}$ are generated in the upper mantle. Geoid anomalies of this wavelength generally have an amplitude of about 20 to 30 meters. An isostatically compensated density anomaly of $0.5 \%$ spread over the upper mantle would give geoid anomalies of this 


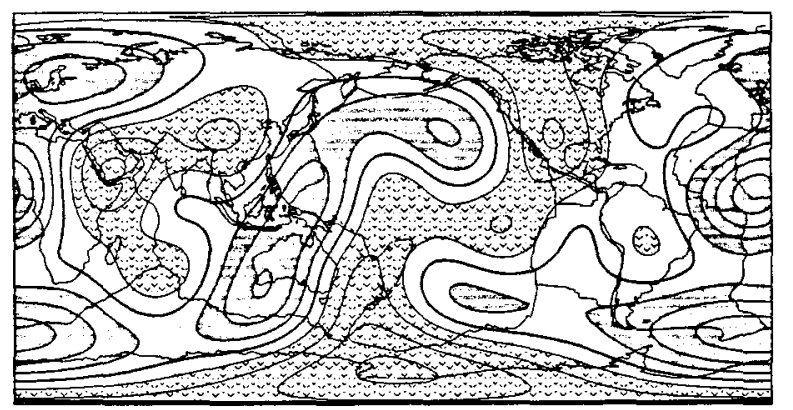

Fig. 1 Phase velocities for $200 \mathrm{sec}$. Rayleigh waves. Spherical harmonics up to $\ell=\mathrm{m}=6$ are included.

size. It therefore appears that a combination of slabs and broad thermal anomalies in the upper mantle can explain the major features of the $\ell=4-9$ geoid. The longer wavelength part of the geoid, $\ell=2-3$, correlates with velocities in the deeper part of the mantle, i.e. lower mantle [Dziewonski et al., 1977; Hager, personal communication] and transition region [Masters et al., 1982; Woodhouse and Dziewonski, 1983]. Fig. 3 shows the actual distribution of Love wave phase velocities and that computed from the geoid assuming a linear relationship between velocity and geoid height. Most subduction regions are slow at short periods, presumably because of backarc basins and hot, upwelling material above the slab.

\section{Azimuthal Anisotropy}

Anisotropy of the upper mantle most likely originates from preferred orientation of olivine crystals. Studies to date indicate that the a-axes of olivine-rich aggregates cluster around the flow direction, the a- and c-axes concentrate in the flow plane, and the b-axes align perpendicular to the flow plane [e.g., Nicolas and Poirier, 1976; Christensen and Salisbury, 1978;] For P-waves the a-, b-, and c-axes are, respectively, the fast, slow, and intermediate velocity directions. If the flow plane is horizontal, the azimuthal P-wave velocity variation is $4 \%$ for natural olivine-rich aggregates [Christensen and Lundquist, 1982]. The corresponding azimuthal SV variation is $2 \%$. This latter value is close to that found in the present study. Rayleigh waves are most sensitive to SV velocities and have less sensitivity to P-waves. The high anisotropy of P-waves, however, means that the azimuthal anisotropy of Rayleigh waves will be affected by the anisotropies of both $\mathbf{P}$ and $S$ waves. The azimuthal variation of both $P$ and $S V$ waves can be described well by a $\cos 2 \theta$ variation, where $\theta$ is measured from the a-axis (fast) direction. The variation of $\mathrm{SH}$ velocity in the flow plane is four-lobed. The fast directions are at $45^{\circ}$ to the a-and c-axes; i.e., the fast direction for Love waves is not parallel to the fast direction for Rayleigh waves. The $\mathrm{SH}$ anisotropy can be expected to be hard to detect, since the variation is small and occurs in only $45^{\circ}$.

For vertical flow, i.e. vertical a-axis, the SV and P anisotropies in the horizontal plane are low for olivine-rich aggregates, the average horizontal $\mathrm{P}$-wave velocity is low, and $\mathrm{SH}<\mathrm{SV}$. The fast direction for $\mathrm{P}$ is in the flow plane, and SV is nearly isotropic. SH has two lobes in the horizontal plane, oriented $45^{\circ}$ to the flow plane. Regions of ascending and descending flow will be characterized by low azimuthal variation
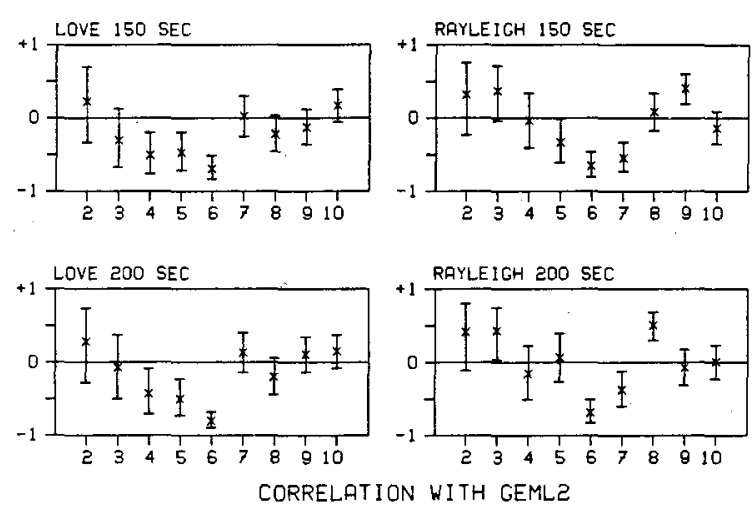

Fig. 2 Correlation coefficients (ordinate) between surface wave phase velocities and the geoid. The horizontal axis gives the order number of the spherical harmonic. Error bars represent $1 \sigma$ range in Fisher's asymptotic theory (Anderson, 1958).

for Rayleigh waves and low Love wave velocities. In regions of descending flow, Rayleigh waves are fast because of low temperatures and the effects of crystal orientation. For both horizontal and vertical flow the nature of the anisotropy is approximately $2 \theta$ for Rayleigh waves, and the fast direction is in the flow plane. For Love waves there is a strong $\cos 4 \theta$ component, and the fast directions are oriented $45^{\circ}$ to the fast directions for Rayleigh waves. Therefore, if olivine orientation controls the anisotropy of the upper mantle, the fast direction of Rayleigh waves should map the flow direction.

Figure 4 is a map of the azimuthal results for $200 \mathrm{~s}$ Rayleigh waves expanded up to $\ell=m=3$. The lines are oriented in the maximum velocity direction, and the length of the lines

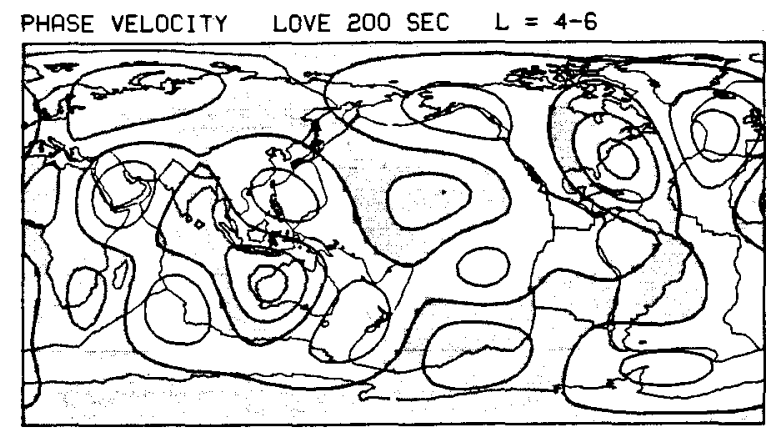

PREDICTED PHASE VELOCITY

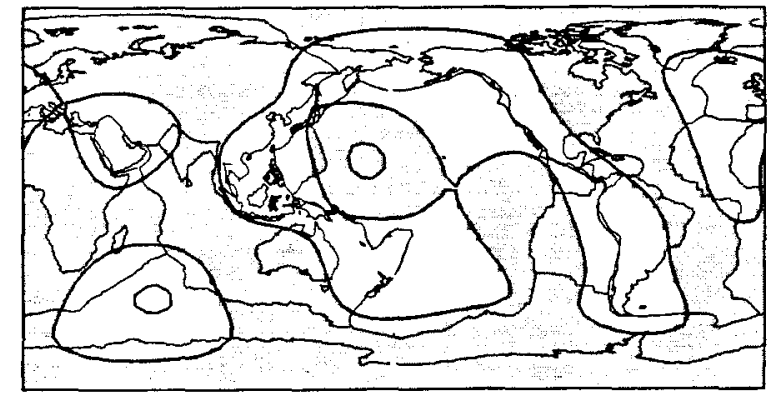

Fig. 3 Love wave phase velocities $(\ell=4-6)$ at $200 \mathrm{sec}$. and phase velocities predicted from a least squares linear fit between phase velocity and geoid height. 
is proportional to the anisotropy. Maximum velocities are oriented approximately NE-SW under Australia, the eastern Indian Ocean, and northern South America and E-Wunder the central Indian Ocean; they vary under the Pacific Ocean from N-S in the southern central region to more NW-SE in the northwest portion. The fast direction is generally perpendicular to plate boundaries. There is little correlation with plate motion directions, and little is expected since these waves are sampling the mantle beneath the lithosphere. Pn velocity correlates well with spreading direction (Bibee and Shor, 1976). The lack of correlation for long-period Rayleigh waves suggests that the flow in the asthenosphere is not strongly coupled to the overlying plate, and this in turn implies a low-viscosity asthenosphere. Hager and O'Connell [1979] have calculated flow in the upper mantle taking into account the drag of the plates and the return flow from subduction zones to spreading centers. Flow lines for a model that includes a low-viscosity channel mantle are shown in Figure 5. The low viscosity under the lithosphere minimizes drag, and the return flow is controlled mainly by the scometry of plate boundaries. Flow under the large fast-moving plates is roughly antiparallel to the plate motions. Thermal buoyancy is ignored in these calculations and there is no lateral variation in viscosity. Hager and O'Connell also calculate shallow mantle flow for a model with a relatively high upper mantle viscosity. In this model there is some coupling between the plate and the underlying mantle; consequently, material is dragged along with the plate. There are major differences in the orientation of flow lines in these two models. The Rayleigh wave fast directions are similar to the flow directions in the low viscosity model but diverge from those of the high viscosity model, particularly under Africa, Australia, India, Greenland, the south Atlantic, and the Tasman Sea. In the kinematic flow model, with a low viscosity asthenosphere, the flow is nearly due south under Australia, shifting to SW under the eastern Indian Ocean; i.e., the return flow is directly from the subduction zones to the ridge. In the anisotropic map the inferred flow is SW under Australia, parallel to the plate motion, and, E-W in the eastern Indian ocean.

There are several possible causes for anisotropy in a convecting mantle. Anisotropic crystals are aligned by flow and by stress. In olivine-rich aggregates the crystals are aligned such that the fast direction is in the flow direction [e.g. Christensen and Salisbury, 1979]. At shallow depths the $\mathrm{Pn}$ velocity is fast in the spreading direction [Bibee and Shor, 1976].

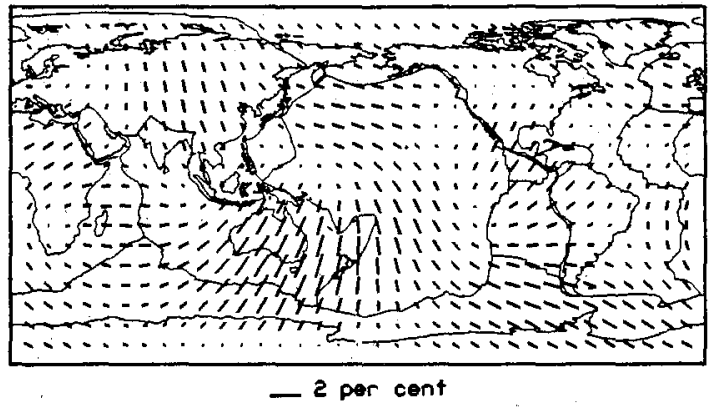

Fig. 4 Azimuthal anisotropy of $200 \mathrm{sec}$. Rayleigh waves. The map includes $\cos 2 \theta$, sin $2 \theta$ and $\ell=1,2$ and 3 terms. The lines indicate the fast phase velocity direction. The length of the lines is proportional to the anisotropy.

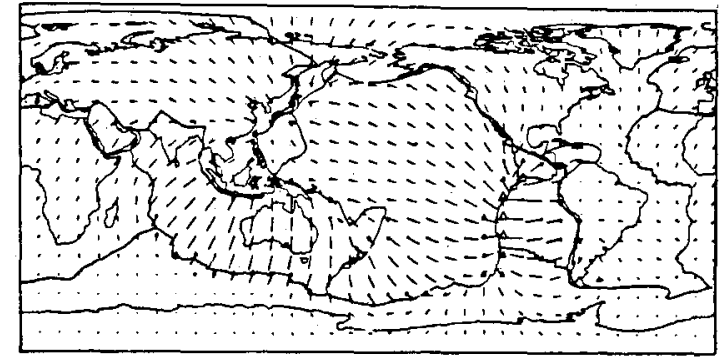

Fig. 5 Flow lines at $260 \mathrm{~km}$ depth for the upper mantle kinematic flow model of Hager and O'Connell (1979). The model includes a low viscosity channel $\left(10^{19}\right.$ poise $)$ in the upper mantle.

Anderson and Regan [1983] found that SH $>$ SV in the upper mantle under the Pacific except near the East Pacific Rise, where ascending flow is likely. These results are consistent with the fast direction being controlled by the flow direction. The good agreement between Figures 4 and 5 suggests that the fast seismic direction does indeed correlate with flow direction. Models with more uniform viscosity [Hager and O'Connell, 1979; Chase, 1979] do not correlate as well, suggesting that low viscosity is a characteristic of the shallow mantle.

The direction of flow in the shallow mantle is fairly independent of the the viscosity model in some regions. For example, the Hager-O'Connell and Chase models give the following orientations: Siberia (NW-SE), North America (NW-SE in the north changing to N-S in the south), Europe (E-W), Nazca plate (E-W), Southern South America (NW-SE), and N.E. Pacific (NW-SE). These are approximately the directions indicated by the seismic anisotropy in these regions. In addition, the low-viscosity model (Model VII of Hager and O'Connell) gives directions in general agreement with the seismic results for much of the Pacific and south Atlantic. Some major differences are apparent which we interpret with the assumption that the fast seismic directions map the flow lines in the mantle. The return flow across the Pacific appears to be affected by the presence of a thermal anomaly near Hawaii. The flow in the north is toward North America, while flow in the south appears to be diverted to the south Pacific. Flow under the southern part of South America is directed toward the south Atlantic, while flow under the northern part of South America is directed toward the nor th Atlantic. The divergence of flow occurs near the Rio Grande Rise. The Indian Ocean Ridge appears to be fed by material subducted under New Guinea and the Sunda Arc.

The direction of flow under Tibet is more N-S than E-W. The flow of the material subducted from the Aleutians and Kamchatka is to the NW rather than directed toward North America. The flow between Kerguelen and the Afar is more $\mathrm{E}-\mathrm{W}$ than N-S. The flow in the vicinity of other hotspots, such as Iceland, Tristan, Azores, Tahiti, and Reunion is different from that predicted by the flow calculations. Thermal buoyancy, including upwelling under hotspots, will affect flow in the mantle and is unaccounted for in the return flow calculations. The extensive low-velocity anomalies near Kerguelen, Red Sea-Afar, Tasman Sea-Tonga, western North America, and the central Pacific may represent upwellings that will alter the return flow patterns calculated from simple 
kinematic considerations. Likewise, the high velocities under western Africa and the south Atlantic may represent dense sinkers, also unaccounted for in the kinematic models. Because of the temperature dependence of viscosity, the flow in these regions may violate the predictions of the models that assume radially symmetric viscosity.

Azimuthal anisotropy of Rayleigh waves appears to be caused by flow in the upper mantle with the fast direction being parallel to the flow lines. The observed azimuthal variation of velocity is consistent with that expected for olivinerich aggregates. There is good agreement between the seismic results and the kinematic upper mantle flow model of Hager and O'Connell [1979] that includes a low viscosity channel. Hotspots appear to affect the flow. Azimuthal anisotropy is low under the North American plate, Europe and the eastern Antarctic plate. There is a good correlation of global surface wave velocities with the $\ell=4-6$ geoid. High velocity areas correlate with geoid lows. Isostatically compensated columns with a density contrast of about $0.5 \%$ spread over about $300 \mathrm{~km}$ can explain the velocity-geoid correlation.

Acknowledgments. We thank Ichiro Nakaniski who kindly provides us with data. Digital data from the SRO and IDA networks made this study possible. We thank Brad Hager for many stimulating discussions and for reviewing the manuscript. This research was supported by NSF grant No. EAR811-5236 and NASA grant No. NSG-7610. Contribution number 4007, Division of Geological and Planetary Sciences, California Institute of Technology, Pasadena, California 91125 .

\section{References}

Anderson, Don L. and J. Regan, Uppermantle anisotropy and the oceanic lithosphere, Geophys. Res. Lett., 10, 841844, 1983.

Anderson, T. W., An Introduction to Multivariate Statistical Analysis, 304 pp., Wiley, New York, 1958.

Bibee L. and G. Shor, Jr., Compressional wave anisotropy in the crust and upper mantle, Geophys. Res. Lett., 3, 639642, 1976.

Chase C., Asthenospheric counterflow: a kinematic model, Geophys. J., 56, 1-18, 1979.

Christensen, N., and S. Lundquist, Pyroxene orientation within the upper mantle, Bull. Geol. Soc. Am., 93, 279288, 1982.

Christensen, N., and M. H. Salisbury, Seismic anisotropy in the oceanic upper mantle, J. Geophys. Res., 84, 46014610, 1979.

Dziewonski, A., B. Hager, and R. O'Connell, Large scale heterogeneities in the lower mantle, J. Geophys. Res. 82, 239-255, 1977.

Hager, B., Global isostatic geoid anomalies for plate and boundary layer models of the lithosphere, Earth Planet. Sci. Lett., 97-109, 1983.

Hager, B., Subducted slabs and the geoid: constraints on mantle rheology and flow, J. Geophys. Res., in press, 1984.

Hager B., and R. O'Connell, Kinematic models of large-scale flow in the Earth's mantle, J. Geophys. Res., 84, 1031$1048,1979$.

Haxby, W. and D. L. Turcotte, On isostatic geoid anomalies, J. Geophys, Res. 83, 5473-5478, 1978.

Masters, G., T. Jordan, P. Silver and F. Gilbert, Aspherical Earth structure from fundamental spheroidal mode data, Nature, 298, 609-613, 1982.

Nakanishi, I. and Don L. Anderson, World-wide distribution of group velocity of mantle Rayleigh waves as determined by spherical harmonic inversion, Bull., Seism. Soc. Am., 72, 1185-1194, 1982.

Nakanishi, I. and Don L. Anderson, Aspherical heterogeneity of the mantle from phase velocities of mantle waves, Nature, 117-121, 1984.

Nataf, H.-C., I. Nakanishi and Don L. Anderson, Anisotropy and shear-velocity heterogeneities in the upper mantle, Geophys. Res. Lett., 11, 109-112, 1984.

Nicolas, A., and J. P. Poirier, Crystalline Plasticity and Solid State Flow in Metamorphic Rocks, John Wiley \& Sons, New York, $437 \mathrm{pp}$.

Tanimoto, T. and Don L. Anderson, Lateral heterogeneity and azimuthal anisotropy of the upper mantle, $J$. Geophys. Res., in press, 1983.

Woodhouse, J. and A. Dziewonski, Mapping the upper mantle, J. Geophys. Res., in press, 1983.

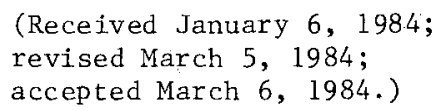

